\title{
Effect of Propranolol on Functional Connectivity in Autism Spectrum Disorder-A Pilot Study
}

\author{
Ananth Narayanan - Catherine A. White • Sanjida Saklayen • Mary J. Scaduto • \\ Allen L. Carpenter • Amir Abduljalil • Petra Schmalbrock • David Q. Beversdorf
}

Published online: 20 May 2010

(C) The Author(s) 2010. This article is published with open access at Springerlink.com

\begin{abstract}
A decrease in interaction between brain regions is observed in individuals with autism spectrum disorder (ASD), which is believed to be related to restricted neural network access in ASD. Propranolol, a beta-adrenergic antagonist, has revealed benefit during performance of tasks involving flexibility of access to networks, a benefit also seen in ASD. Our goal was to determine the effect of propranolol on functional connectivity in ASD during a verbal decision making task as compared to nadolol, thereby accounting for the potential spurious fMRI effects due to peripheral hemodynamic effects of propranolol. Ten ASD subjects underwent fMRI scans after administration of placebo, propranolol or nadolol, while performing a phonological decision making task. Comparison of functional connectivity between pre-defined ROI-pairs revealed
\end{abstract}

Dr. Beversdorf has spoken for Pfizer, Eisai and Novartis in the past, unrelated to this work.

\footnotetext{
A. Narayanan $\cdot$ S. Saklayen

Integrated Biomedical Sciences Graduate Program,

The Ohio State University,

Columbus, OH, USA
}

\author{
A. Narayanan $\cdot$ S. Saklayen $\cdot$ D. Q. Beversdorf $(\bowtie)$ \\ Department of Radiology, \\ University of Missouri, Thompson Center, \\ 300 Portland St., Suite 122, \\ Columbia, MO 65211, USA \\ e-mail: beversdorfd@health.missouri.edu \\ C. A. White \\ Departments of Psychiatry and Neurology, \\ The Ohio State University, \\ Columbus, $\mathrm{OH}$, USA \\ S. Saklayen · M. J. Scaduto \\ College of Medicine, The Ohio State University, \\ Columbus, OH, USA
}

a significant increase with propranolol compared to nadolol, suggesting a potential imaging marker for the cognitive effects of propranolol in ASD.

Keywords Autism · Functional connectivity . Noradrenergic $\cdot$ Language · Propranolol .

Asperger syndrome

\section{Introduction}

Autism is a neurodevelopmental disorder characterized by impaired social interaction, impaired communication and repetitive and restricted behaviors (Lord et al. 1994). While varying degrees of mental retardation are common in
A. L. Carpenter

Neuroscience Graduate Studies Program,

The Ohio State University,

Columbus, OH, USA

A. Abduljalil · P. Schmalbrock

Department of Radiology, The Ohio State University,

Columbus, OH, USA

D. Q. Beversdorf

Department of Neurology, University of Missouri, Columbia, MO, USA

D. Q. Beversdorf

Department of Psychology, University of Missouri,

Columbia, MO, USA 
autism (Lord et al. 1994), even those without global cognitive impairment encounter significant problems trying to function in society (Morgan 1996; Carpenter 1992). Autism spectrum disorder (ASD) is a term used to describe the spectrum of autism, Asperger syndrome and pervasive developmental disorder-not otherwise specified (Beversdorf et al. 1998).

Several theories have been proposed to explain the impairments in autism, including inability to comprehend the perspectives of others ("theory of mind") (Baron-Cohen et al. 1985), inability to process emotional information (Hobson 1991, 1993; Fotheringham 1991) and impaired executive function (Rumsey 1985; Rumsey and Hamburger 1988, 1990). Individuals with autism are also known to have decreased central coherence, or difficulty with utilization of context to process its environmental relevance (Frith and Happé 1994; Happé 1994). This lack of central coherence is believed be related to restriction of semantic and associative networks, leading to the observed deficits in semantic clustering in verbal memory (Minshew and Goldstein 2001). This also relates to superior performance in recognition on "false memory" tasks (Beversdorf et al. 2000 ), proposed to be associated with increased neuronal density and decreased neuronal size in the hippocampus, among other atypical findings in the brain in autism (Bauman and Kemper 1985, 1994).

More recently, a potential neural correlate of this phenomenon of network underconnectivity and decreased context utilization in autism has been revealed, with the demonstration of decreased functional connectivity, defined as the 'temporal correlation between spatially remote neurophysiological events' (Friston 1994). This is demonstrated by decreased functional connectivity fMRI (fcMRI) during sentence comprehension and working memory in high functioning individuals with autism (Just et al. 2004), and during a range of other cognitive tasks (Koshino et al. 2005).

Our recent pilot evidence has suggested a benefit in flexibility of access to networks on verbal problem solving tasks with propranolol in ASD (Beversdorf et al. 2008). In this study, subjects with ASD solved simple anagram tasks, which involved a search through the lexical/semantic network to find the solution (for example, 'BRICK' is the solution to 'IRBCK'), more quickly after administration of propranolol than after placebo. Therefore, we wished to determine whether propranolol might also result in an increase in functional connectivity in ASD.

Propranolol, a $\beta$-adrenergic antagonist, appears to affect flexibility of access to lexical, semantic and associative networks on verbal problem solving tasks in individuals without neurodevelopmental diagnoses (Beversdorf et al. 1999). In the initial work, anagram performance was better after propranolol, a $\beta$-adrenergic antagonist, as compared to ephedrine, an adrenergic agonist (Beversdorf et al. 1999).
In subsequent work, anagram performance was found to be significantly better after propranolol, which blocks both central and peripheral $\beta$-adrenergic receptors, as compared to nadolol, which blocks only peripheral $\beta$-adrenergic receptors, suggesting that noradrenergic modulation of cognitive flexibility is mediated by a central, rather than a peripheral mechanism (Beversdorf et al. 2002). However, in neither of these initial small studies in individuals without developmental diagnoses did the improvement with propranolol as compared to placebo reach significance. In order to better understand the effect of propranolol, a subsequent larger study was performed, which revealed a significant benefit from propranolol for the subjects slowest at solving the problems, as well as for the most difficult problems regardless of subject (Campbell et al. 2008). However, benefit was not observed for subjects best at solving the problems, or for simple problems regardless of subject, with a decline in performance occasionally observed with propranolol in these cases. This was true for anagrams as well as other verbal problem solving tasks requiring flexibility of access to lexical, semantic and associative networks. In contrast to the studies in individuals without neurodevelopmental diagnoses, in the study examining the effect of propranolol in ASD a benefit was observed for the simplest anagrams with propranolol as compared to placebo, despite a slight decline in performance with the same tasks with propranolol among control participants (Beversdorf et al. 2008).

The effect of propranolol is believed to be due to the modulatory effect of norepinephrine on the signal-tonoise ratio (SNR) of neuronal activity within the cortex (Hasselmo et al. 1997). Increased SNR would be expected to increase the relative strength of dominant responses, but decreased SNR may allow greater access to more remote associative inputs, which may benefit cognitive flexibility in unconstrained tasks such as those involving search of semantic and associative networks (Hasselmo et al. 1997; Alexander et al. 2007). This is consistent with the aforementioned finding where propranolol benefitted solving difficult verbal problem solving tasks, but not simple tasks, in individuals without neurodevelopmental diagnoses (Campbell et al. 2008). In addition, propranolol was shown to reverse the cognitive impairment induced by stress (Alexander et al. 2007) and acute cocaine withdrawal (Kelley et al. 2007), during which the observed deleterious effects on cognitive performance is believed to be due to noradrenergic upregulation, regardless of task difficulty.

Both social and language benefits have been reported with $\beta$-blockers in a pilot case series study in autism (Ratey et al. 1987). Furthermore, our previous evidence suggests a beneficial effect of propranolol on verbal problem solving in ASD (Beversdorf et al. 2008). While it is unclear whether noradrenergic activity is dysregulated in autism 
(Martchek et al. 2006; Minderaa et al. 1994), we wished to explore the effects of propranolol on functional connectivity between brain regions during a language task, because of these observed cognitive effects of the drug. However, propranolol is also utilized for lowering blood pressure and heart rate. Since fMRI's blood oxygenation level dependent (BOLD) signal is heavily dependent on the hemodynamic response, likely to be affected by changes in heart rate and blood pressure, the effects of propranolol will be compared to that of nadolol. Nadolol does not pass through the bloodbrain barrier while propranolol does, and therefore nadolol has the same peripheral effects without any of the central effects of propranolol. This will help to ensure that any effect on functional connectivity is not an epiphenomenon of the peripheral hemodynamic effects of propranolol. As propranolol will block peripheral vascular receptors as well as central receptors, any indirect central effects secondary to peripheral effects of propranolol would be controlled for with the propranolol vs. nadolol comparison. Therefore, we predicted that functional connectivity would be greater after propranolol than after nadolol, which would serve as our primary outcome measure.

Thus, in order to address this hypothesis, we examined the effect of these beta-adrenergic antagonists on connectivity in individuals with ASD during a well characterized word categorization task (McDermott et al. 2003), with which performance is at ceiling in individuals with normal intelligence, and for which phonological categorization demonstrated a high degree of connectivity within language areas in individuals without neurodevelopmental diagnoses in previous studies (Tivarus et al. 2008). In general, selective impairment in individuals with ASD occurs in conditions which place elevated demands on information processing (Williams et al. 2006), which manifests as a decrease in utilization of contextual information in processing of semantic information (Minshew and Goldstein 2001; Beversdorf et al. 2000). However, impairments in phonological information processing are also observed in ASD (Schmidt et al. 2008). Phonological categorization was utilized in this study since decreased connectivity between distant cortical regions in ASD appears to occur across a range of tasks (Just et al. 2004; Koshino et al. 2005), and since high connectivity observed in unaffected individuals with the phonological categorization task (Tivarus et al. 2008), it was expected that this task would be sensitive to the predicted connectivity enhancing effects of the adrenergic agents. A phonological task was also selected since the effects of propranolol on tasks involving searches of the phonological network were sometimes greater than for searches involving the semantic network in our previous work in individuals without neurodevelopmental diagnoses (Campbell et al. 2008) and in individuals acutely withdrawing from cocaine (Kelley et al. 2007).

\section{Methods}

Research participants

Ten right-handed, native-English speaking adults ( 8 males), aged 19-29 (mean $24.3 \pm 4.37$ std dev), with a mean FSIQ of $102.89 \pm 14.0$ and an average of $12.33 \pm 0.8$ years of education, diagnosed with ASD using the ADI-R (Autism Diagnostic Interview-Revised) (Lord et al. 1994) criteria were recruited to participate in this study. In addition, all subjects were diagnosed with the GADS (Gilliam Asperger's Disorder Scale) (Gilliam 2001) criteria for Asperger syndrome. Two of the subjects had a diagnosis of autism, and the other eight had a diagnosis of Asperger Syndrome. Of the eight with Asperger Syndrome, two were on the borderline ADI-R criteria for the Communication Subscale in addition to being above criteria on the other subscales. Subjects with a history of dyslexia, cardiac disease, asthma, diabetes or thyroid disease were excluded, as were subjects with any recent history of noradrenergic drug use. All subjects were able to comprehend the task and perform the task outside the scanner. Exclusion criteria also included a history of comorbid psychiatric disorders such as schizophrenia, major depression, bipolar disorder or major head trauma. One subject had a history of seizures, treated with felbamate and lamotrigine. Two had been given the diagnosis of attention deficit disorder in the past, but were not actively treated for this. Two had been given the diagnosis of obsessive compulsive disorder, and one among the two had also been diagnosed with generalized anxiety disorder. One subject was taking sertraline and one was taking fluoxetine, but no other psychotropics were taken by the participants. No changes had been made in the dosages of these medications in at least 6 months before the study. All participants either reported normal vision, or were provided corrective lenses in order for them to view the fMRI stimuli. Participants were also screened to comply with MRI safety requirements (no metallic implants or prostheses, no metal objects in their bodies, nonclaustrophobic). Written consent was obtained from all study participants after explaining the study and the nature of the procedure to each participant, in accordance with the regulations of the Institutional Review Board of The Ohio State University.

\section{Drug administration}

All participants were required to attend one test session for each drug, with the visits being at least $24 \mathrm{~h}$ apart. During each visit, the participant was orally administered either placebo, propranolol $(40 \mathrm{mg})$, or nadolol $(50 \mathrm{mg})$. The $50 \mathrm{mg}$ dose of nadolol was utilized since it exhibited identical effects on heart rate as the $40 \mathrm{mg}$ dose of 
propranolol in our previous work (Beversdorf et al. 2002). Propranolol is rapidly absorbed from the small intestine and reaches peak plasma levels in 1-2 $\mathrm{h}$ after oral dose, and nadolol, 2-4 $\mathrm{h}$ after the dose. The timing was also that used in previous experiments, which revealed cognitive effects (Beversdorf et al. 2002). Therefore, imaging was initiated $60 \mathrm{~min}$ after propranolol and placebo, and $120 \mathrm{~min}$ after nadolol. In order to avoid learning effects, the order of drug administration was counterbalanced, and the subjects, imaging staff and analysis team were blinded to drug condition. Heart rate and blood pressure were measured before administration of the drug, and immediately before and after the fMRI scans.

\section{Materials}

Separate groups of word-lists for phonological processing were created for each session by modifying previously used stimuli (McDermott et al. 2003), which have demonstrated robust activation of phonological language areas, and revealed a high degree of functional connectivity between language areas in healthy controls (Tivarus et al. 2008). Each group consisted of four word-lists, for a total of 12 lists presented to each participant over the course of the entire experiment (Tivarus et al. 2008).

Every participant performed one version of the phonological task during each visit. The task was a block design task (Fig. 1) with 4 task blocks (24 s), each interspersed between 5 rest blocks $(30 \mathrm{~s})$ for a total of $4 \mathrm{~min}$ and $6 \mathrm{~s}$. During each task block, a cue word was presented in capitalized letters ( $3 \mathrm{~s})$, followed by the corresponding word-list $(1.1 \mathrm{~s}$ for each word followed by a $300 \mathrm{~ms}$ timeout). A '*' sign was shown during the rest blocks. Presentation and recording was done using E-Prime version 1.1 and a RF-interference free LCD monitor placed inside the scanner room. The subject was instructed to attend to the sound of each word in the word list, and respond whether it rhymed with the cue word presented at the beginning of the block, using an fMRI-compatible response system (Lumina LP 400, Cedrus Corp., San Pedro, CA).

Imaging data acquisition and analysis

Images were collected with a 3T Philips scanner with an 8channel SENSE head coil. Structural T1-weighted images were acquired using a T1 weighted $3 \mathrm{D}$ FFE pulse sequence $(\mathrm{TR}=25 \mathrm{~ms}$; TE$=3.6 \mathrm{~ms} ; 512 \times 512$ matrix; $240 \mathrm{~mm}$ FOV; 64 axial slices; $2.2 \mathrm{~mm}$ thick). BOLD contrast functional scans were acquired using a gradient echo EPI sequence $(\mathrm{TR}=3 \mathrm{~s} ; \mathrm{TE}=35 \mathrm{~ms} ; 80 \times 80$ matrix; $230 \mathrm{~mm}$ FOV; 35 axial slices, $4 \mathrm{~mm}$ thick; $\alpha=90^{\circ}$ ). The first two images were acquired to allow stabilization of longitudinal magnetization and not included in further analysis. The imaging data were analyzed using SPM8. Each BOLD series was corrected for slice acquisition timing, motion corrected for respiratory and other motion artifacts, normalized to the Montreal Neurological Institute (MNI) template, and resampled and smoothed using $5 \mathrm{~mm}$ Gaussian kernel to decrease spatial noise.

Statistical analysis was performed on individual data using the general linear model as implemented in SPM8. Average group activation maps were generated using one sample $t$-tests and between drug difference images using two-sample $t$-tests as implemented in SPM8. Functional connectivity was measured as the correlation between the average time series of different ROI-pairs. Four ROIs known to be activated by the phonological task (McDermott et al. 2003), including the left inferior frontal cortex (BA 44/45/ 46)-LIFG, left fusiform gyrus (BA37)-LFUS, left parietal cortex (BA7)-LPAR and left middle temporal gyrus (BA 21/ 22)-LMTG, were selected a priori. Spherical $(10 \mathrm{~mm}$ diameter) ROIs centered within the abovementioned regions were drawn and confirmed by a fellowship trained Behavioral and Cognitive Neurologist in the Departments of Radiology and Neurology (D.Q.B.), on the standard template. Average time series for all voxels included in the ROI were extracted for each participant for each drug condition using MATLAB. Correlations of the time series between pairs of ROIs were computed by calculating the correlation coefficient between the time series for each ROI pair for each subject for each drug condition. Fisher's Ztransformation was applied to the computed correlations for each a priori ROI-pair to enable comparison between drug conditions and ROI-pairs.

\section{Results}

Axial slices of average group activation maps generated through SPM5 revealed a pattern of activity similar to previous studies (McDermott et al. 2003; Tivarus et al. 2008) (Fig. 2), including a set of brain regions comprised of the LIFG extending to the premotor and motor areas, bilateral middle frontal gyrus, left posterior middle temporal gyrus, left fusiform gyrus, bilateral occipital cortex and bilateral premotor cortex. There was no significant difference detected for any of the between-drug comparisons in any of the a priori language-related ROIs. Errors were rare on the task, with no subject responding with less than $88 \%$ correct under any condition, and there were no differences in task accuracy between drug conditions.

Repeated measures $2 * 6$ ANOVA (drug*ROI-pair) showed a main effect of significantly greater connectivity $[F(1,7)=7.742 ; P=0.027]$ with propranolol (mean $r=0.457$; $\mathrm{SE}=0.09$ ) as compared to nadolol (mean $r=0.321 ; \mathrm{SE}=0.10$ ) (Fig. 3). Further analysis also revealed a drug*ROI-pair 
Fig. 1 Task design. Task design for one phonological task presented in this experiment. In the example shown, the words brand, land and sand rhyme with the cue word, STAND, while the word clips does not

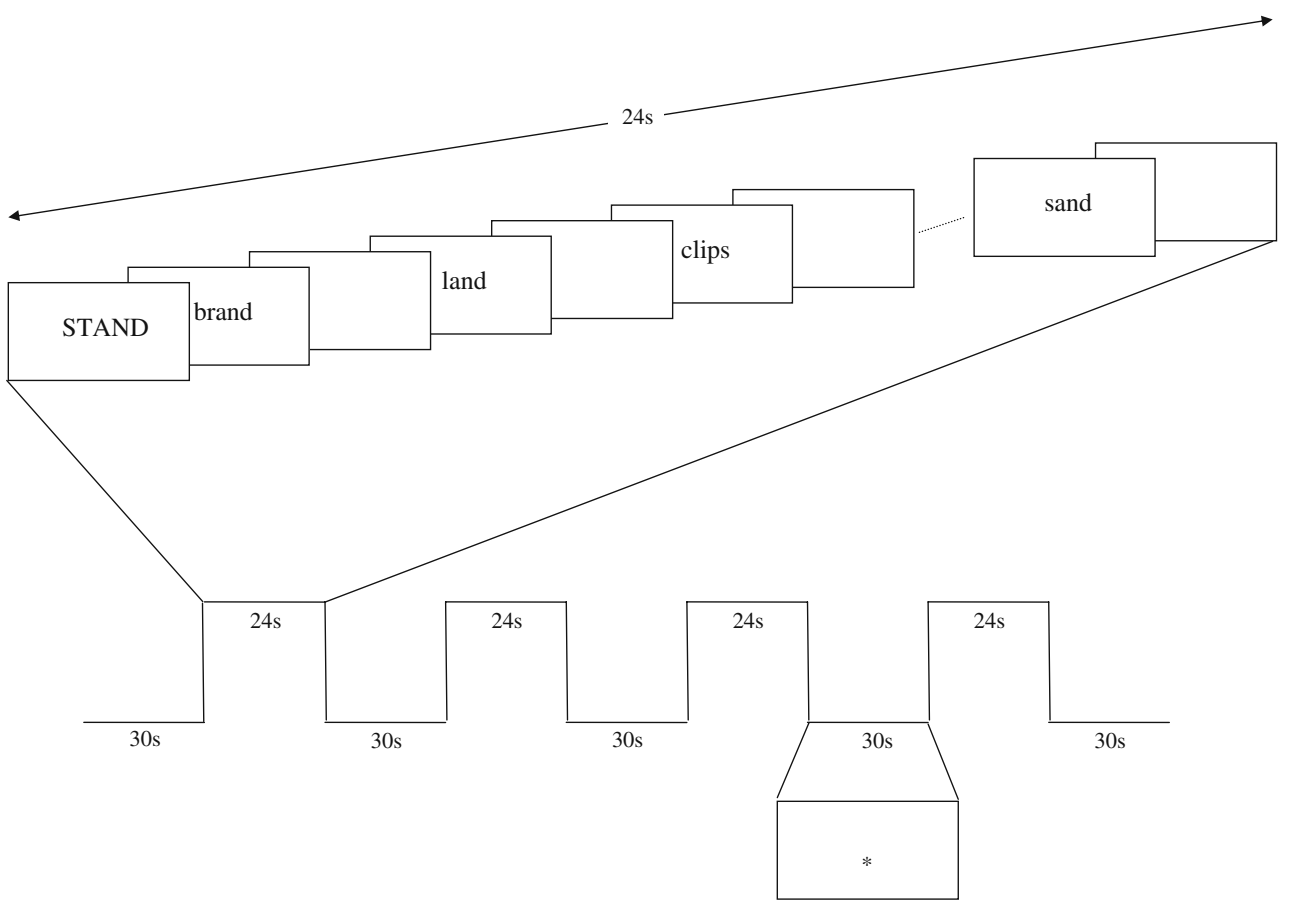

interaction $[F(1,7)=33.029 ; P=0.008]$ and a trend towards main effect of ROI-pair $[F(1,7)=7.140 ; P=0.068]$. Analysis of individual ROI-pairs revealed significantly $[t(7)=4.263$; $\mathrm{x}$ $p=0.004]$ greater functional connectivity between LIFG and LFUS after propranolol (mean $r=0.4029$; $\mathrm{SE}=0.04$ ) than after nadolol (mean $r=0.1123$; $\mathrm{SE}=0.07$ ), which withstood Bonferroni correction. In a similar comparison between these ROI-pairs, the main effect for the comparison between propranolol and placebo (mean $r=0.344$; $\mathrm{SE}=0.11$ ) did not reach significance (Fig. 3), and no main effect for ROI pair or interaction effect was found for this comparison.

There was no main effect for a difference in connectivity between placebo and nadolol, and also no main effect for ROI pair or interaction effect was found for this comparison.

Both heart rate (HR) and systolic blood pressure (SBP) significantly decreased in a similar manner from baseline to pre fMRI assessment for propranolol (paired $t$-tests- SBP: $p=0.017$, HR: $p=0.009$ ) and nadolol (SBP $p=0.006$, HR: $p=0.013)$ and from baseline to post fMRI assessment for propranolol (SBP: $p=0.026$, HR: $p=0.011$ ) and nadolol (SBP: $p=0.020$, HR: $p=0.0002$ ), and no significant change was observed with placebo for any of these conditions. There was no significant difference in HR or SBP between the post-drug pre fMRI assessments and post fMRI assessments for placebo, propranolol, or nadolol.

\section{Discussion}

These results show that propranolol appears to increase the functional connectivity between brain regions in ASD as compared to nadolol. This is consistent with our hypothesis, since propranolol, with central and peripheral $\beta$ adrenergic blockade, shows increased connectivity as compared to the condition where only peripheral $\beta$ adrenergic receptors are blocked. The adequacy of the nadolol as a control for the peripheral $\beta$-adrenergic effects is supported since blood pressure and heart rate effects were similar between nadolol and propranolol.

These results may begin to suggest an anatomic substrate of the cognitive effects of propranolol previously reported in ASD (Beversdorf et al. 2008). However, while this task was chosen due to its ability to elicit a high degree of functional connectivity in previous studies (Tivarus et al. 2008), it was not designed for concurrent assessment of performance. Therefore, exploring the effect of the noradrenergic system on functional connectivity in both controls and individuals with ASD, in tasks that are known to be modulated by the noradrenergic system and that allow for concurrent performance monitoring is critical for better understanding of the modulatory effect of the noradrenergic system. Recent evidence from diffusion tensor imaging (DTI) has suggested that the decreased functional connectivity observed in autism is, at least in part, anatomically based (Lee et al. 2007). However, our study suggests that at least part of this decrease in connectivity can be manipulated pharmacologically. Based upon the proposed effects of the noradrenergic system on SNR (Hasselmo et al. 1997), the increase in functional connectivity between distant brain regions with propranolol as compared to nadolol may be an observable manifestation of the increase in access to more remote associative inputs, which would 
Fig. 2 Group activation maps. Average group activation maps during phonological processing among individuals with autism after administration of placebo (a), propranolol (b), and nadolol (c). The left portion of each demonstrates the Maximum Intensity Projection (MIP) generated through SPM8 (presented in neurological convention)
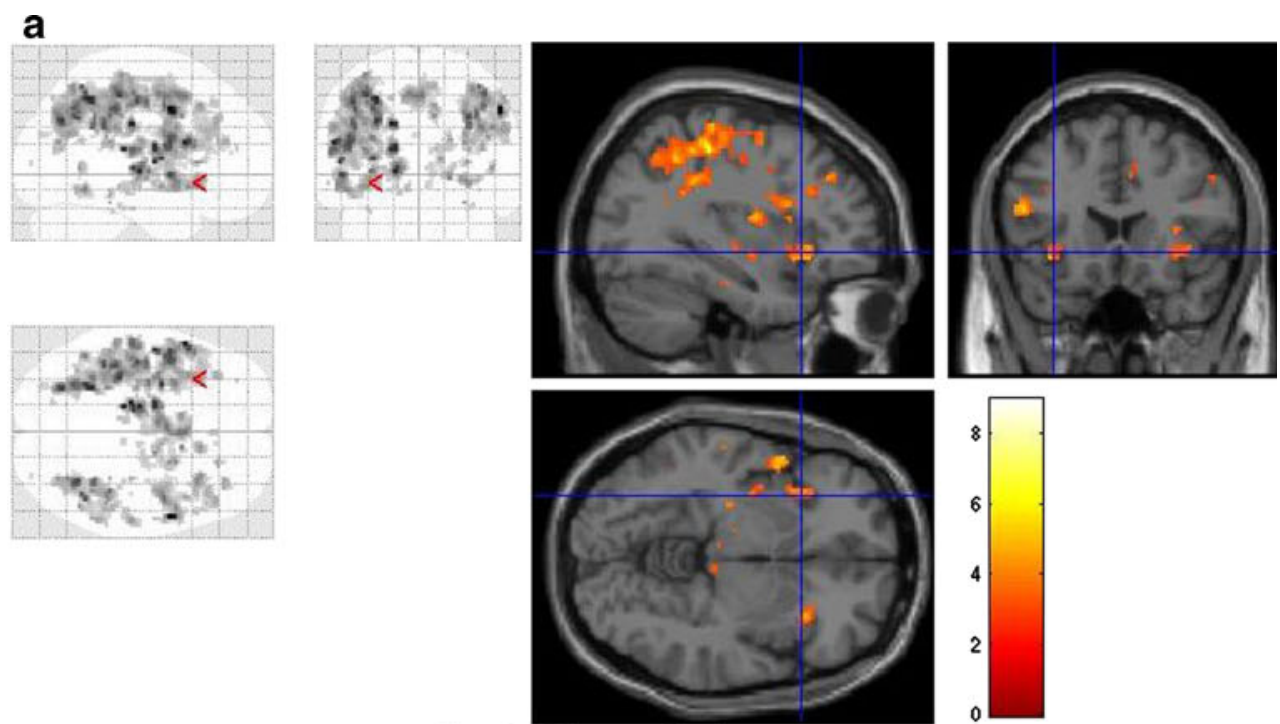

Placebo Phono > Rest

b
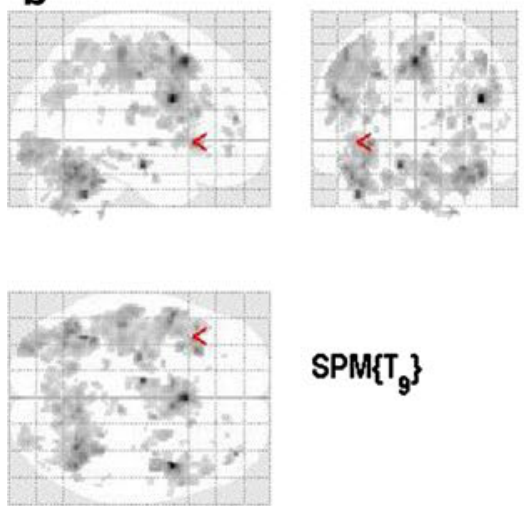

SPM $\left\{T_{9}\right\}$
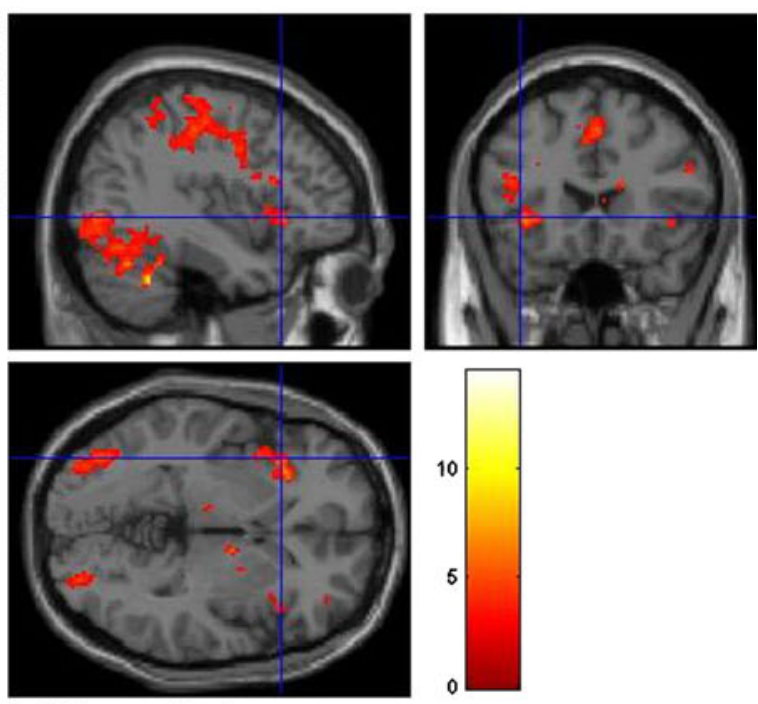

Propranolol Phono > Rest

C
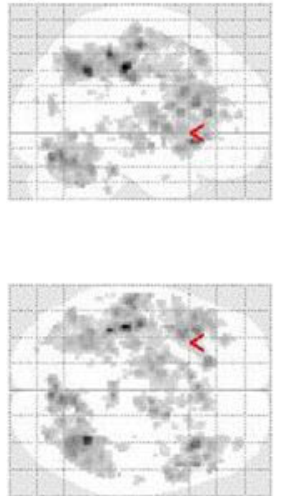

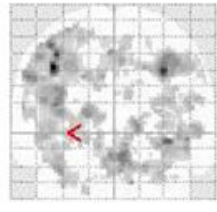

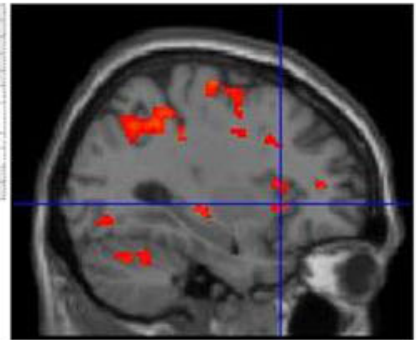
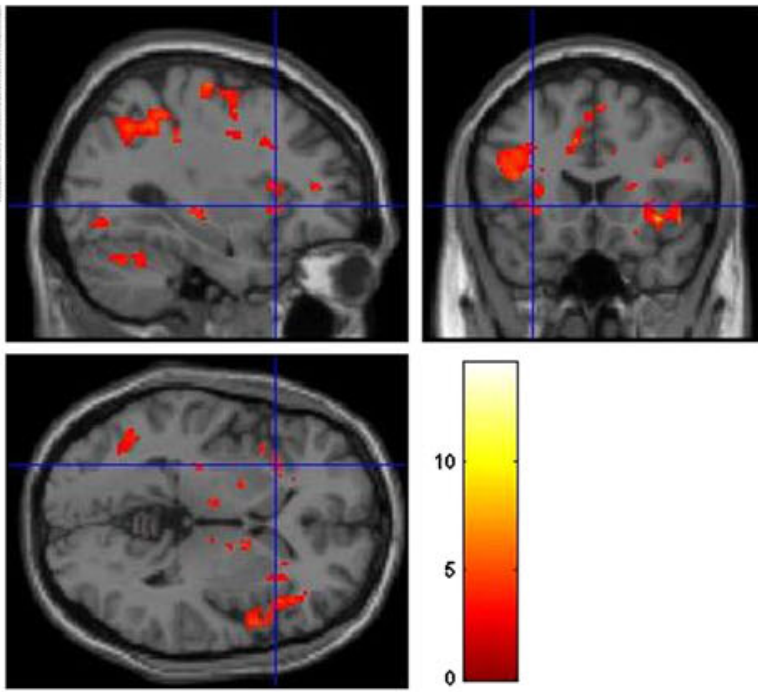

Nadolol Phono > Rest 


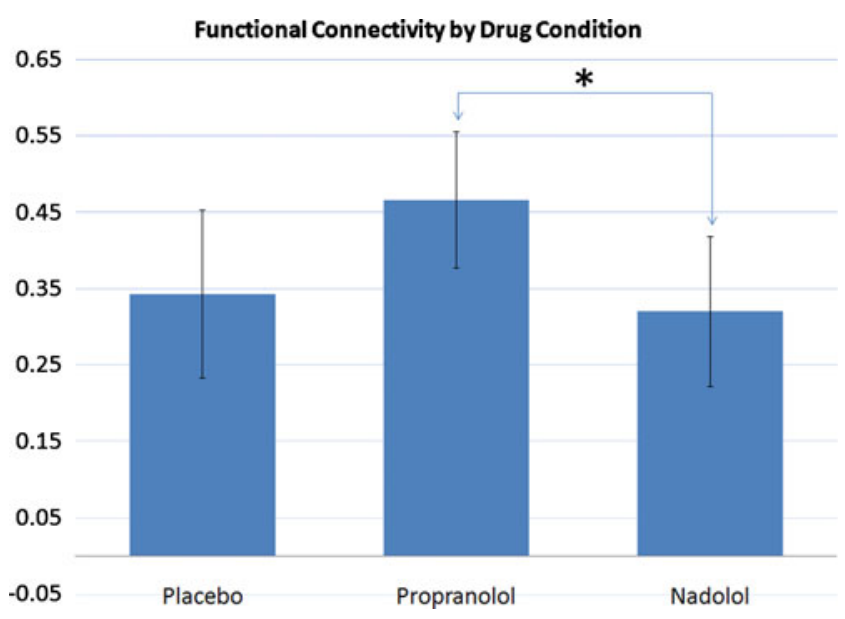

Fig. 3 Comparison of correlation coefficients. Mean correlation coefficients (average over all ROI-pairs) for the phonological task for each of the drug conditions

benefit cognitive flexibility in unconstrained tasks such as searches of lexical/semantic/associative networks, associated with decreased noradrenergic activity (Hasselmo et al. 1997; Alexander et al. 2007). Further study examining tasks more sensitive to pharmacological effects on performance would be necessary to better understand the relationship between performance and connectivity.

Whereas it was not the primary focus of this study, no significant difference was observed between propranolol and placebo. This may be due to the fact that the addition of other hemodynamic factors, such as differences in blood pressure between the drug conditions, interfered with our ability to detect a difference between propranolol and placebo. Specifically, propranolol decreases blood pressure and heart rate as compared to placebo, which would likely also impact the BOLD response in fMRI. Therefore, any comparison between propranolol and placebo would also be confounded by other differences in BOLD effects. By comparing propranolol to nadolol, the blood pressure and heart rate effects are matched between drug conditions, minimizing any confounding effects of peripheral hemodynamics on the BOLD response, and giving a more specific indication of the central effects of propranolol.

The small size of this study limits our ability to draw any firm conclusions, though, regarding the comparison between propranolol and placebo. Larger studies will be needed to further evaluate the imaging effects of propranolol as compared to placebo, and the relationship between the imaging effects and these hemodynamic factors. This further investigation of the propranolol and placebo comparison will be critical for better understanding of any future clinical implications of this study. Also, our study focused on a priori regions of interest, but future studies will also need to examine the role of the right hemisphere due to atypical functional lateralization of language reported in ASD (Kleinhans et al. 2008). Furthermore, the specific finding of increased connectivity between LIFG and LFUS with propranolol as compared to nadolol is of interest in light of the role of the LIFG in decision making in language tasks (Bookheimer 2002) and the role of the LFUS in visual word form recognition (Beversdorf et al. 1997). This suggests a specific role of propranolol in the interaction between visual word recognition and language decision making in phonological decision making. However, additional study is necessary to further address this, including the relationship between drug effects on connectivity and on task performance.

Others have proposed a role of the noradrenergic system in autism. Based on research revealing that individuals with autism can actually have improved behaviors and communication during febrile episodes (Curran et al. 2007), it has been proposed that fever may transiently restore adrenergic tone in a developmentally dysregulated and hypofunctioning adrenergic system in autism (Mehler and Purpura 2009). Regardless of whether noradrenergic activity is actually altered or not in ASD (Martchek et al. 2006; Minderaa et al. 1994), the decreased flexibility of access to phonological, semantic and associative networks in autism (Schmidt et al. 2008; Beversdorf et al. 2000), and the effects of propranolol on flexibility of access to such networks (Campbell et al. 2008), would be consistent with the imaging findings observed in this study, as well as the cognitive effects in autism (Beversdorf et al. 2008). Regardless of whether norepinephrine is upregulated, as has been proposed (Lake et al. 1977; Launay et al. 1987), or downregulated (Mehler and Purpura 2009), this points to the relevance of understanding noradrenergic activity in ASD, as it appears to be important. This also points to the importance of examining both higher and lower functioning subjects, as our findings have revealed benefits from the beta-adrenergic antagonist propranolol in high functioning subjects (Beversdorf et al. 2008), but many of the subjects in the Curran et al. (2007) study revealing the beneficial effects of fever, upon which the hypotheses of Mehler and Purpura (2009) were based, were lower functioning individuals with autism. Furthermore, our study predominantly examined patients with Asperger Syndrome, where language is relatively preserved. Future studies will need to determine whether benefits also occur in the autism patient population, whether higher or lower functioning. Inclusion of controls without neurodevelopmental diagnoses will also be necessary in future studies to better understand the effect of propranolol on functional connectivity, and whether this effect is specific to ASD. Also, the highly selective sample in this study limits the generalizability of any conclusions that can be drawn from our findings.

Better converging evidence from neuropsychopharmacological studies and neuroimaging research in autism may 
ultimately help guide future potential supportive treatments for autism, if appropriate biomarkers can be identified. Furthermore, clinical trials will also be needed in order to better understand the clinical implications of this finding. Also, both propranolol and nadolol are nonselective $\beta$ adrenergic antagonists. The distinct roles of beta- 1 and beta-2 receptors in this effect will need to be determined in future studies. Considerable effort has been undertaken to better understand the differences between cognitive effects of alpha and beta adrenergic receptors (for example, Chamberlain et al. 2006), but less is known about the differential effects of agents acting on beta- 1 and beta- 2 receptors. However, given recent work revealing the apparently opposing effects of beta- 1 and beta- 2 receptors on working memory, with improved working memory in animal models with the beta-1 adrenergic antagonist, betaxolol (Ramos et al. 2005), but improved working memory in animal models with the beta-2 agonist, clenbuterol (Ramos et al. 2008), better understanding of the role of the specific beta receptors will be critical for further investigation of the clinical implications of noradrenergic-based treatment of ASD.

\begin{abstract}
Acknowledgement This research is funded through a pilot grant from National Alliance for Autism Research (now Autism Speaks) (1033/DB//01-201-005-00-00), grants from NINDS (K23 NS43222), The Ohio State University Medical Center Research Investment Fund and the Wright Center for Innovation, and by the University of Missouri Department of Radiology Research Investment Fund. We would like to thank Bradley Ferguson for his help with the manuscript.
\end{abstract}

Open Access This article is distributed under the terms of the Creative Commons Attribution Noncommercial License which permits any noncommercial use, distribution, and reproduction in any medium, provided the original author(s) and source are credited.

\section{References}

Alexander, J. K., Hillier, A., Smith, R. M., Tivarus, M. E., \& Beversdorf, D. Q. (2007). Beta-adrenergic modulation of cognitive flexibility during stress. Journal of Cognitive Neuroscience, 19(3), 468-478, [Pubmed: 17335395].

Baron-Cohen, S., Leslie, A. M., \& Frith, U. (1985). Does the autistic child have a 'theory of mind'? Cognition, 21(1), 37-46, [Pubmed: 2934210].

Bauman, M., \& Kemper, T. L. (1985). Histoanatomic observations of the brain in early infantile autism. Neurology, 35(6), 866-874, [Pubmed: 4000488].

Bauman, M., \& Kemper, T. (1994). The neurobiology of autism. Baltimore: Johns Hopkins.

Beversdorf, D. Q., Ratcliffe, N. R., Rhodes, C. H., \& Reeves, A. G. (1997). Pure alexia: clinical-pathologic evidence for a lateralized visual language association cortex. Clinical Neuropathology, 16 (6), 328-331, [Pubmed: 9401800].

Beversdorf, D. Q., Anderson, J. M., Manning, S. E., Anderson, S. L., Nordgren, R. E., Felopulos, G. J., et al. (1998). The effect of semantic and emotional context on written recall for verbal language in high functioning adults with autism spectrum disorder. Journal of Neurology, Neurosurgery, and Psychiatry, 65(5), 685-692, [Pubmed: 9810938].

Beversdorf, D. Q., Hughes, J. D., Steinberg, B. A., Lewis, L. D., \& Heilman, K. M. (1999). Noradrenergic modulation of cognitive flexibility in problem solving. NeuroReport, 10(13), 2763-2767, [Pubmed: 10511436].

Beversdorf, D. Q., Smith, B. W., Crucian, G. P., Anderson, J. M., Keillor, J. M., Barrett, A. M., et al. (2000). Increased discrimination of "false memories" in autism spectrum disorder. Proceedings of the National Academy of Sciences of the United States of America, 97(15), 8734-8737, [Pubmed: 10900024].

Beversdorf, D. Q., White, D. M., Chever, D. C., Hughes, J. D., \& Bornstein, R. A. (2002). Central beta-adrenergic blockers modulation of cognitive flexibility. NeuroReport, 13(18), 25052507, [Pubmed: 12499857].

Beversdorf, D. Q., Carpenter, A. L., Miller, R. F., Cios, J. S., \& Hillier, A. (2008). Effect of propranolol on verbal problem solving in autism spectrum disorder. Neurocase, 14(4), 378-383, [Pubmed: 18766980].

Bookheimer, S. (2002). Functional MRI of language: new approaches to understanding the cortical organization of semantic processing. Annual Review of Neuroscience, 25, 151-188, [Pubmed: 12052907].

Campbell, H. L., Tivarus, M. E., Hillier, A., \& Beversdorf, D. Q. (2008). Increased task difficulty results in greater impact of noradrenergic modulation of cognitive flexibility. Pharmacology, Biochemistry and Behavior, 88(3), 222-229, [Pubmed: 17884154].

Carpenter, A. (1992). Autistic adulthood: A challenging journey. In E. Schopler \& G. B. Mesibov (Eds.), High-functioning individuals with autism (pp. 289-294). New York: Plenum.

Chamberlain, S. R., Müller, U., Blackwell, A. D., Robbins, T. W., \& Sahakian, B. J. (2006). Noradrenergic modulation of working memory and emotional memory in humans. Psychopharmacology, 188, 397-407, [Pubmed 16642355].

Curran, L. K., Newschaffer, C. J., Lee, L. C., Crawford, S. O., Johnston, M. V., \& Zimmerman, A. W. (2007). Behaviors associated with fever in children with autism spectrum disorders. Pediatrics, 120(6), e1386-e1392, [Pubmed: 18055656].

Fotheringham, J. B. (1991). Autism: Its primary psychological and neurological deficit. Canadian Journal of Psychiatry, 36(9), 686692, [Pubmed: 1773407].

Friston, K. J. (1994). Functional and effective connectivity in neuroimaging: a synthesis. Human Brain Mapping, 2, 56-78.

Frith, U., \& Happé, F. G. E. (1994). Autism: Beyond "theory of mind". Cognition, 50(1-3), 115-132, [Pubmed: 8039356].

Gilliam, J. E. (2001). Gilliam Asperger's disorder scale. Austin: ProEd.

Happé, F. G. E. (1994). An advanced test of theory of mind: understanding of story characters' thoughts and feelings by able autistic, mentally handicapped, and normal children and adults. Journal of Autism and Developmental Disorders, 24(2), 129154, [Pubmed: 8040158].

Hasselmo, M. E., Linster, C., Patil, M., Ma, D., \& Cekic, M. (1997). Noradrenergic suppression of synaptic transmission may influence cortical signal-to-noise ratio. Journal of Neurophysiology, 77(6), 3326-3339, [Pubmed: 9212278].

Hobson, R. P. (1991). Methodological issues for experiments on autistic individuals' perception and understanding of emotion. Journal of Child Psychology and Psychiatry, 32(7), 1135-1158, [Pubmed: 1787140].

Hobson, R. P. (1993). Understanding persons: The role of affect. In S. Baron-Cohen, H. Tager-Flusberg, \& D. J. Cohen (Eds.), Understanding other minds: Perspectives from autism (pp. 208-227). Oxford: Oxford University Press.

Just, M. A., Cherkassky, V. L., Keller, T. A., \& Minshew, N. J. (2004). Cortical activation and synchronization during sentence compre- 
hension in high-functioning autism: evidence of underconnectivity. Brain, 127(8), 1811-1821, [Pubmed: 15215213].

Kelley, B. J., Yeager, K. R., Pepper, T. H., Bornstein, R. A., \& Beversdorf, D. Q. (2007). The effect of propranolol on cognitive flexibility and memory in acute cocaine withdrawal. Neurocase, 13(5), 320-327, [Pubmed: 18781429].

Kleinhans, N. M., Muller, R. A., Cohen, D. N., \& Courchesne, E. (2008). Atypical functional lateralization of language in autism spectrum disorders. Brain Research, 1221, 115-125, [Pubmed: 18555209].

Koshino, H., Carpenter, P. A., Minshew, N. J., Cherkassky, V. L., Keller, T. A., \& Just, M. A. (2005). Functional connectivity in an fMRI working memory task in high-functioning autism. Neuroimage, 24(3), 810-821, [Pubmed: 15652316].

Lake, C. R., Ziegler, M. G., \& Murphy, D. L. (1977). Increased norepinephrine levels and decreased dopamine-beta-hydroxylase activity in primary autism. Archives of General Psychiatry, 34, 553-556, [Pubmed: 558741].

Launay, J.-M., Bursztejn, C., Ferrari, P., Dreux, C., Braconnier, A., Zarifian, E., et al. (1987). Catecholamines metabolism in infantile autism: a controlled study of 22 autistic children. Journal of Autism and Developmental Disorders, 17, 333-347, [Pubmed: 3654486].

Lee, J. E., Bigler, E. D., Alexander, A. L., Lazar, M., DuBray, M. B., Chung, M. K., et al. (2007). Diffusion tensor imaging of white matter in the superior temporal gyrus and temporal stem in autism. Neuroscience Letters, 424(2), 127-132, [Pubmed: 17714869].

Lord, C., Rutter, M., \& Le Couteur, A. (1994). Autism Diagnostic Interview-Revised: a revised version of a diagnostic interview for caregivers of individuals with possible pervasive developmental disorders. Journal of Autism and Developmental Disorders, 24 (5), 659-685, [Pubmed: 7814313].

Martchek, M., Thevarkunnel, S., Bauman, M., Blatt, G., \& Kemper, T. (2006). Lack of evidence of neuropathology in the locus coeruleus in autism. Acta Neuropathologica, 111(5), 497-499, [Pubmed: 16596425].

McDermott, K. B., Petersen, S. E., Watson, J. M., \& Ojemann, J. G. (2003). A procedure for identifying regions preferentially activated by attention to semantic and phonological relations using functional magnetic resonance imaging. Neuropsychologia, 41(3), 293-303, [Pubmed: 12457755].

Mehler, M. F., \& Purpura, D. P. (2009). Autism, fever, epigenetics and the locus coeruleus. Brain Research Reviews, 59(2), 388-392, [Pubmed: 19059284].

Minderaa, R. B., Anderson, G. M., Volkmar, F. R., Akkerhuis, G. W., \& Cohen, D. J. (1994). Noradrenergic and adrenergic functioning in autism. Biological Psychiatry, 36(4), 237-241, [Pubmed: 7986888].
Minshew, N. J., \& Goldstein, G. (2001). The pattern of intact and impaired memory functions in autism. Journal of Child Psychology and Psychiatry, 42(8), 1095-1101, [Pubmed: 11806691].

Morgan, H. (1996). Underpinning philosophy in the provision of services for adults with autism: A critique of global values related to specific practice. In H. Morgan (Ed.), Adults with autism: A guide to theory and practice (pp. 31-52). Cambridge: Cambridge University Press.

Ramos, B. P., Colgan, L., Nou, E., Ovadia, S., Wilson, S. R., \& Arnsten, A. F. T. (2005). The beta-1 adrenergic antagonist, betaxolol, improves working memory performance in rats and monkeys. Biological Psychiatry, 58, 894-900, [Pubmed: 16043136].

Ramos, B. P., Colgan, L. A., Nou, E., \& Arnsten, A. F. T. (2008). $\beta 2$ adrenergic agonist, clenbuterol, enhances working memory performance in aging animals. Neurobiology of Aging, 29, 1060-1069, [Pubmed: 17363115].

Ratey, J. J., Bemporad, J., Sorgi, P., Bick, P., Polakoff, S., O’Driscoll, G., et al. (1987). Open trial effects of beta-blockers on speech and social behaviors in 8 autistic adults. Journal of Autism and Developmental Disorders, 17(3), 439-446, [Pubmed: 3654495].

Rumsey, J. M. (1985). Conceptual problem-solving in highly verbal, nonretarded autistic men. Journal of Autism and Developmental Disorders, 15(1), 23-36, [Pubmed: 3980427].

Rumsey, J. M., \& Hamburger, S. D. (1988). Neuropsychological findings in high-functioning men with infantile autism, residual state. Journal of Clinical and Experimental Neuropsychology, 10 (2), 201-221, [Pubmed: 3350920].

Rumsey, J. M., \& Hamburger, S. D. (1990). Neuropsychological divergence of high-level autism and severe dyslexia. Journal of Autism and Developmental Disorders, 20(2), 155-168, [Pubmed: 2347817].

Schmidt, G. L., Kimel, L. K., Winterrowd, E., Pennington, B. F., Hepburn, S. L., \& Rojas, D. C. (2008). Impairments in phonological processing and nonverbal intellectual function in parents of children with autism. Journal of Clinical and Experimental Neuropsychology, 30(5), 557-567, [Pubmed: 18569254].

Tivarus, M. E., Hillier, A., Schmalbrock, P., \& Beversdorf, D. Q. (2008). Functional connectivity in an fMRI study of semantic and phonological processes and the effect of 1-Dopa. Brain and Language, 104(1), 42-50, [Pubmed: 17418888].

Williams, D. L., Goldstein, G., \& Minshew, N. J. (2006). Neuropsychologic functioning in children with autism: further evidence for disordered complex information processing. Child Neuropsychology, 12, 279-298, [Pubmed: 16911973]. 